\title{
Terahertz transistors based on aligned carbon nanotube arrays
}

\author{
Li DING \& Zhiyong ZHANG*
}

Key Laboratory for the Physics and Chemistry of Nanodevices and Department of Electronics and Center
for Carbon-based Electronics, Department of Electronics, Peking University, Beijing 100871, China

Received 13 October 2021/Accepted 26 October 2021/Published online 3 November 2021

Citation Ding L, Zhang Z Y. Terahertz transistors based on aligned carbon nanotube arrays. Sci China Inf Sci, 2022, 65(1): 117301, https://doi.org/10.1007/s11432-021-3355-0

The wireless communication technology has greatly promoted the industry's demand for higher operating frequencies and wider bandwidth devices. In recent years, the rise of $5 \mathrm{G}$ and $6 \mathrm{G}$ communication technologies needs to increase the operating frequency of devices to a frequency band above $90 \mathrm{GHz}$. In addition, the development of the semiconductor industry also urgently needs chips with high operating frequencies and integration. Two most commonly used kinds of RF devices are silicon-based CMOS technology (complementary metal oxide semiconductor) and III-V compound semiconductor technology (mainly GaAs, InP, etc.). Silicon-based CMOS technologies can achieve high integration at low cost, but their linearity and noise performance are poor at frequencies above $20 \mathrm{GHz}$. Devices based on III-V compounds can be used to build systems with operating frequencies exceeding $20 \mathrm{GHz}$, but they cannot be directly integrated with digital systems on the same chip. An ideal general-purpose semiconductor radio frequency chip should have both high operating frequency and high integration while it can be used to design not only radio frequency/analog circuits for communication and radar applications but also digital integrated circuits for information processing purposes. Therefore, new principles, new materials, and new device structures are needed to promote the further development of communication technology.

Semiconductor carbon nanotubes have electrical characteristics such as ultra-high carrier mobility and saturation speed, excellent intrinsic linearity, and small intrinsic size, high chemical stability, good thermal conductivity, and high mechanical strength. They are used in both digital integrated circuits and radio frequency circuits with great potential and advantage of constructing radio frequency/digital circuit $\mathrm{SoC}$ system integration platform which is expected to provide ideal chip for even future $6 \mathrm{G}$ communication technology.

Recently, the research group of Zhang and Peng from Peking University developed semiconductor carbon nanotube array materials suitable for radio frequency applications through improved self-assembly and dual phase deposition method. Based on the carbon nanotube array, they realized for the first-time radio frequency transistors and high-performance amplifiers that are expected to work in the terahertz band [1], fully demonstrating the advantages and potential of carbon nanotubes in radio frequency electronics. They also optimized the material preparation for the large-scale radio frequency application. The PCO-Bpy molecule with fewer alkyl chains was subjected to two-time dispersion purification and two-liquid phase self-assembly deposition process to achieve a high-semiconductor purity on a 4-inch wafer with high purity ( $>99.99 \%)$, high orien-

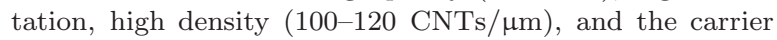
mobility up to $1580 \mathrm{~cm}^{2} \cdot \mathrm{V}^{-1} \cdot \mathrm{s}^{-1}$. The research group fabricated carbon nanotube array radio frequency transistors on a fully insulated quartz substrate with the measured cut-off frequency $\left(f_{\mathrm{T}, \mathrm{EXT}}\right)$ and power gain cut-off frequency $\left(f_{\mathrm{MAX}, \mathrm{EXT}}\right)$ of the 50 -nm gate length device reached 186 and $158 \mathrm{GHz}$ respectively which are the highest values of all transistors based on nanomaterials. Transistors fabricated on high-resistance silicon substrates exhibit up to 1.9 $\mathrm{mA} / \mu \mathrm{m}$ on-state current and $1.4 \mathrm{mS} / \mu \mathrm{m}$ transconductance (when $V_{\mathrm{ds}}=-0.9 \mathrm{~V}$ ). The intrinsic characteristics of 50$\mathrm{nm}$ gate length $\mathrm{RF}$ transistors with $540 \mathrm{GHz}$ cut-off frequency $\left(f_{\mathrm{T}, \mathrm{INT}}\right)$ and $306 \mathrm{GHz}$ power gain cut-off frequency $\left(f_{\mathrm{MAX}, \mathrm{INT}}\right)$ indicate that carbon-based radio frequency devices have entered the terahertz band for the first time. They further explored the potential application of a carbonbased radio-frequency amplifier, by using a multi-finger gate structure to boost the load driving capability to achieve the carbon-based amplifier with higher gain and linearity. For the first time, working at $18 \mathrm{GHz}$ (K band), carbon-based amplifiers achieve a gain of up to $23.2 \mathrm{~dB}, 1 \mathrm{~dB}$ compression point output power over $9 \mathrm{dBm}$ and a third-order intermodulation characteristic of OIP3 over $31 \mathrm{dBm}$ while the corresponding OIP $3 / P_{\mathrm{dc}}$ is $19.7 \mathrm{~dB}$. All achieved key metrics are much higher than the best carbon-based radio frequency transistors or amplifiers previously reported and even comparable with III-V compound semiconductor technology.

\section{References}

1 Shi H, Ding L, Zhong D, et al. Radiofrequency transistors based on aligned carbon nanotube arrays. Nat Electron, 2021, 4: 405-415

\footnotetext{
* Corresponding author (email: zyzhang@pku.edu.cn)
} 